\title{
Modificaciones Hemodinámicas Asociadas a la Asimetría de Aneurismas de Aorta Abdominal y su Influencia en las Posibilidades de Ruptura
}

\author{
Félix Nieto $^{(1)}$, Guillermo Vilalta ${ }^{(2) *}$, María Á. Peréz ${ }^{(1,3)}$, Laurentiu M. Lipsa ${ }^{(2)}$ \\ (1) Departamento CMIM, EGI, ICGF, IM e IPF, Escuelas de Ingenierías Industriales, Universidad \\ de Valladolid, Calle Paseo del Cauce, 59, 47011, Valladolid, España. (e-mail: felnie@eis.uva.es) \\ (2) CARTIF Centro tecnológico, Área de IPF, Parque Tecnológico de Boecillo, P. 205, 47 151, \\ Boecillo, España. (e-mail: guivil@cartif.es; liplau@cartif.es) \\ (3) Instituto de las Tecnologías Avanzadas de la Producción-ITAP, Universidad de Valladolid, Calle \\ Paseo del Cauce, 59, 47011, Valladolid, España. (e-mail: marper@eis.uva.es) \\ ${ }^{*}$ Autor a quien dirigir la correspondencia
}

Recibido Sep. 23, 2011; Aceptado Nov. 10, 2011; Versión final recibida Nov. 15, 2011

\section{Resumen}

Se analiza la influencia de la asimetría de los aneurismas de aorta abdominal en la dinámica del flujo sanguíneo en el interior del saco aneurismático y se discute cómo este comportamiento puede influir en el potencial de ruptura de los aneurismas. Para ello se realiza un estudio numérico empleando cuatro modelos geométricos idealizados con diferente grado de asimetría. La sangre es modelada como Newtoniana, homogénea, incompresible y laminar con propiedades semejantes a los valores que típicamente se emplean en la literatura. Los resultados mostraron que el flujo en el interior del saco aneurismático es altamente perturbado con intensas estructuras de vórtices que viajan desde el extremo proximal al distal, ejerciendo una gran influencia en la distribución superficial de las tensiones tangenciales. También se ha establecido que, a pesar que los aneurismas son asimétricos, este factor tiene un peso ponderado bajo en las posibilidades de ruptura de los aneurismas.

Palabras clave: aneurismas de aorta abdominal, AAA, asimetría de aneurismas, ruptura de aneurismas

\section{Hemodynamic Modifications Associated to Abdominal Aortic Aneurysm Asymmetry and its Influence on Rupture Potential}

\begin{abstract}
Influence of abdominal aortic aneurysms asymmetry on the hemodynamics within the aneurysmatic sac is analyzed and its influence on the rupture potential is discussed. A numerical study by using four idealized geometric models, with different asymmetry degrees, is carried out. Blood is modelled as a newtonian, homogeneous, incompressible and laminar flow with properties as typically used in the literature. The results showed that the flow inside of the aneurysmatic sac is highly disturbed with intense vortex structures travelling from proximal to distal ends, strongly influencing on the wall shear stress distribution. It have also been established that, despite that the aneurysm are asymmetric, this factor has low weight on aneurysm rupture.
\end{abstract}

Keywords: abdominal aortic aneurysms, AAA, aneurysm asymmetry, aneurysm rupture 


\section{INTRODUCCIÓN}

El proceso evolutivo de los aneurismas de aorta abdominal (AAAs) es un complejo proceso donde intervienen múltiples factores relacionados, fundamentalmente, con la remodelación destructiva de la pared arterial. Bajo ciertas condiciones específicas y aún no comprendidas en su totalidad, el aneurisma tiende a incrementar de tamaño con riesgo de ruptura lo cual, de ocurrir, causaría hemorragia interna, con altos índices de mortalidad y elevados costos asociados fundamentalmente a la cirugía de urgencia y al tratamiento de recuperación de los pacientes. Los aneurismas suelen ser asintomáticos, debido a lo cual la ruptura ocurre sin "aviso previo", lo que valoriza cualquier esfuerzo que se realice en el sentido de identificar un criterio fiable asociado con el riesgo de ruptura real de un aneurisma sobre bases personalizadas.

Actualmente, desde el punto de vista clínico los indicadores utilizados para evaluar el riesgo de ruptura de un aneurisma son el diámetro máximo transversal y su tasa de crecimiento. Cuando el diámetro máximo es del orden de $5-5.5 \mathrm{~cm}$ y/o el aneurisma crece más de $0.5 \mathrm{~cm} / a n ̃ o$ (independientemente del tamaño), el paciente es sometido a uno de los dos tratamientos de reparación electiva habituales: reparación endovascular del aneurisma (EVAR, por su siglas en inglés) o reparación por injerto (open surgery). En caso contrario se mantiene bajo observación, sometido a chequeos periódicos. Sin embargo, existe consenso que estos indicadores no son precisos y en no pocas ocasiones fallan. Por ejemplo, (Fillinger et al., 2003) establecen que entre un $10-24 \%$ de pequeños aneurismas (< $<\mathrm{cm}$ de diámetro máximo) rompen. Por otro lado, (Papaharilaou et al., 2007) publican un estudio de un aneurisma de $10 \mathrm{~cm}$ de diámetro, caso este, totalmente inusual desde el punto de vista clínico.

Debido a esta limitación, en los últimos años se está realizando un intenso esfuerzo de investigación para la obtención de un modelo que permita mejorar la fiabilidad en la evaluación precisa del riesgo de ruptura (Gasser et al., 2010, Maier et al., 2010, McGloughlin y Doyle, 2010, Ene et al., 2011). Este novedoso enfoque se basa en la determinación de relaciones funcionales entre factores de diferentes naturalezas (biológicas, estructurales y geométricas) y escalas (temporales y dimensionales) a niveles molecular, celular, de tejido y de órgano, que permita describir desde el punto de vista cuantitativo la evolución de los aneurismas.

Dentro de estos determinantes, uno de los más explorados en la actualidad es el relacionado con la geometría de los AAA, ya que ha sido comprobado que su importancia es absolutamente relevante en la evaluación del riesgo de ruptura. En general, el aneurisma puede ser definido como una deformación progresiva y localizada de la aorta, siendo el grado de deformación un indicador del estado de desarrollo de esta patología, lo define desde el punto de vista geométrico y está bien documentado que esta forma tiene una gran influencia en el potencial de ruptura (Vilalta et al., 2010). Los principales parámetros geométricos que caracterizan el aneurisma, y que pueden ser determinados fácilmente a través de la TAC, son: diámetro máximo transversal $D$, diámetro de la arteria aorta infrarenal $d$, la longitud $L$, el diámetro de la luz arterial $D_{L}$ y el espesor de pared $t$. Otros parámetros pueden ser derivados de estos: $L_{A}$ es la longitud anterior y $L_{P}$ es definida como longitud posterior. Por otro lado, recientemente (Giannoglou et al. 2006, Georgakaratos et al. 2010) se han definido otros parámetros geométricos, pero su determinación se realiza a través de complejos procedimientos matemáticos, lo que dificulta el objetivo de utilizar estos determinantes geométricos como "simples" predictores de ruptura.

Los parámetros geométricos han sido convenientemente relacionado para definir los factores biomecánicos de naturaleza geométrica (Kleinstreuer y Li, 2006, Vilalta et al., 2011a): tasa de deformación, asimetría, índice de dilatación, espesor relativo, razón área AAA/Trombo intraluminal y tasa de crecimiento.

Los aneurismas son esencialmente asimétricos debido a la restricción al movimiento impuesta por las vértebras lumbares. La propia definición de la asimetría de los aneurismas condiciona la existencia de una superficie aneurismal muy compleja que ejerce una gran influencia en las principales variables asociadas a la integridad estructural de la pared y consecuentemente sobre las posibilidades de ruptura (Finol et al., 2003).

Atendiendo a estos aspectos, en el presente trabajo se estudia la influencia de la asimetría de los aneurismas de aorta abdominal, en la dinámica del flujo sanguíneo en el interior del saco 
aneurismático y cómo este comportamiento puede influir en el potencial de ruptura de los aneurismas.

\section{MÉTODOS Y MATERIALES}

\section{Dominio computacional}

Durante la realización del presente trabajo, se utilizaron 4 modelos geométricos idealizados de aneurismas de aorta abdominal, los cuales fueron generados en CATIA V.5R19 (Dessault Systèmes, Paris). Para la generación de estos modelos, y como método para la validación del modelo computacional, fue utilizado el método propuesto por Scotti (Scotti et al., 2005), donde se define el grado de asimetría $\beta$, según la Ecuación 1:

$$
\beta=\frac{r}{R}
$$

siendo $r$ y $R$ los radios medidos en la sección media del saco aneurismático, desde el eje axial $(z)$ entre las paredes posterior y anterior respectivamente. El dominio que representa el fluido está caracterizado por una sección circular paralela al plano $x y$, cuyo diámetro representativo de la entrada y salida es constante e igual a $d=2 \mathrm{~cm}$ y por un diámetro máximo de $D=3 d$, ubicado en la sección media del saco aneurismático, como se muestra en la Figura 1, para un valor de asimetría de 0.2. Una característica importante y que debe tenerse presente a la hora del análisis de los resultados, es que los aneurismas representados por estos modelos presentan un diámetro máximo que está en el rango adecuado para ser sometido a una reparación quirúrgica.

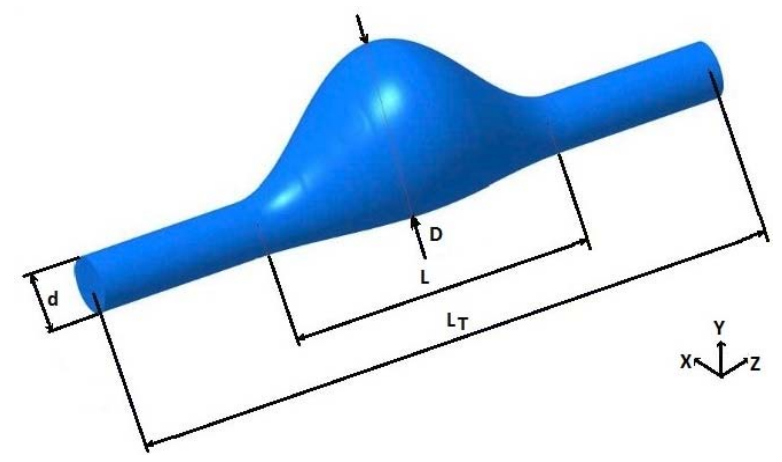

Fig. 1: Representación del modelo geométrico de AAA, con grado de asimetría $\beta=0.2$.

\section{Ecuaciones de gobierno y condiciones de contorno}

Las ecuaciones de gobierno para el dominio del fluido, son las ecuaciones de continuidad y de Navier-Stokes, a las que se le aplicaron las siguientes consideraciones: homogeneidad, incompresibilidad, comportamiento Newtoniano y pulsátil en ausencia de fuerzas de campo. Es conocido que la sangre es una suspensión no Newtoniana de células en plasma, pero es razonable modelarla como fluido Newtoniano en vasos mayores de, aproximadamente, $0.5 \mathrm{~mm}$ de diámetro (Milnor 1989). Las propiedades físicas de la sangre utilizadas fueron aquellas de reconocida validez por la literatura: densidad, $\rho=1050 \mathrm{~kg} / \mathrm{m}^{3}$ y coeficiente de viscosidad dinámica, $\mu=0,004$ Pa.s. Estas ecuaciones, en su forma compacta se representan por las Ecuaciones 2 y 3 .

$$
\begin{gathered}
. u=0 \\
\frac{D u}{D t}=-\frac{1}{\rho} \quad p+v \quad{ }^{2} u
\end{gathered}
$$

donde $\boldsymbol{u}$ es el vector velocidad y $p$ es la presión.

El pulso de onda de velocidad usado en el presente estudio es mostrado en la Figura 2. 


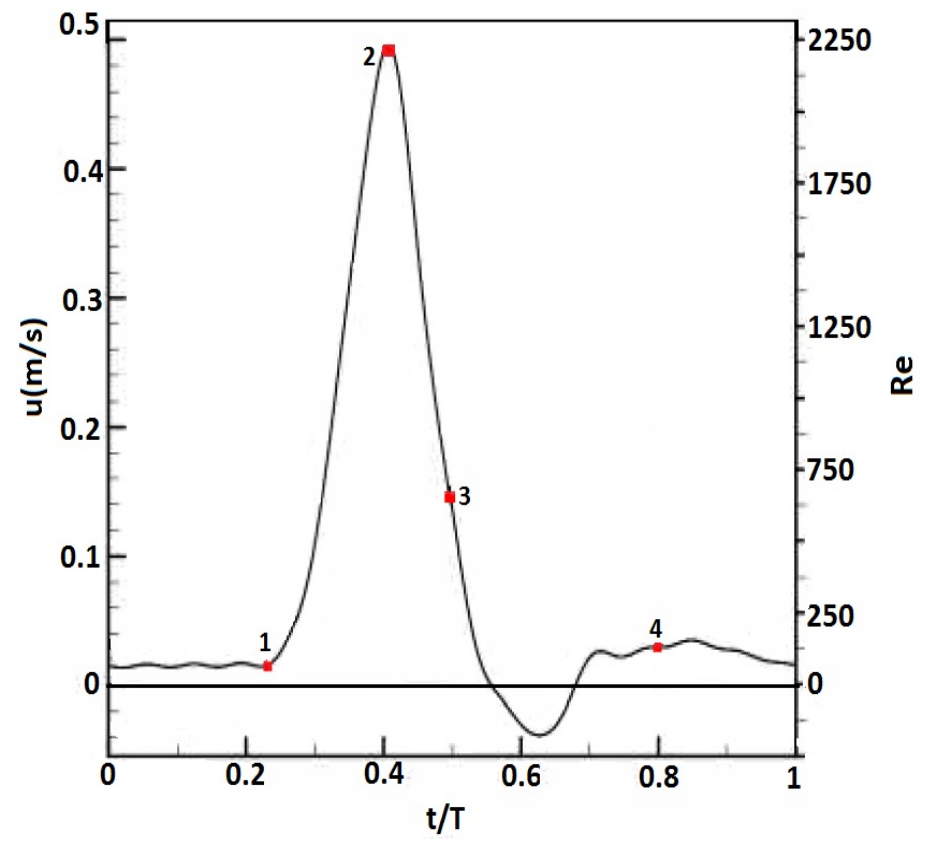

Fig. 2: Pulso en velocidad utilizado en el presente estudio.

Esta función temporal, está dada por representaciones en series de Fourier, de la función que se expresa por la Ecuación (4)

$$
f(t)=A_{0}+\sum_{k=1}^{N}\left(A_{k} \cos 2 \pi k t+B_{k} \operatorname{sen} 2 \pi k t\right)
$$

donde $N$ es el número de armónicos utilizados para reproducir in vivo las mediciones de la velocidad en el segmento aórtico $u(t)$, siendo en este caso $N=18$. Estas funciones son pulsos trifásicos apropiados para condiciones hemodinámicas normales en el segmento infrarenal de la arteria aorta humana, como ha sido reportado por Mills et al (1970). El uso de un pulso transitorio a la entrada basado en una condición fisiológicamente normal, se justifica por el hecho de que la condición de contorno en la entrada es aplicada por encima del cuello proximal del aneurisma, un segmento no dilatado de la arteria aorta. Como se conoce, en condiciones de reposo, el flujo sanguíneo en la arteria aorta es generalmente laminar (Finol \& Amon, 2002), la desaceleración del flujo que se alcanza después del máximo sistólico induce al establecimiento de un flujo laminar localmente perturbado y a la formación de vórtices (Moore \& Ku, 1994). Para el pulso utilizado, el máximo sistólico se obtiene, aproximadamente, en $\mathrm{t}=0.4 \mathrm{~s}$. El valor del número adimensional de Reynolds $R e$, es del orden de 410 , basado en el valor medio de la velocidad y el diámetro en la sección de entrada. Por otro lado, el número de Womersley $\alpha$, que caracteriza la relación entre la frecuencia del flujo ( $T=1 \mathrm{~s}$ ), la geometría y las propiedades físicas de la sangre, es 12.2 , que es un valor típico para el segmento aórtico en estudio.

Las condiciones de contorno impuestas para la modelación pulsátil fueron:

Velocidad: perfil desarrollado en la sección de entrada y condición de no deslizamiento en las paredes. Estas condiciones se representan por el conjunto de las Ecuaciones (5)

Presión: Libre de tensiones en la región de salida, caracterizado por la Ecuación (6), debido a lo cual, los resultados obtenidos están referidos a este valor,

$$
\begin{aligned}
& W(t)=2(u(t))\left(1-\frac{\left(x^{2}+y^{2}\right)}{R a^{2}}\right) ; U=V=0 \\
& \tau_{i j}=-p \delta_{i j}+\mu\left(\frac{\partial u_{i}}{\partial x_{j}}+\frac{\partial u_{j}}{\partial x_{i}}\right)=0
\end{aligned}
$$




\section{Método numérico}

Las ecuaciones de gobierno son resueltas utilizando el software ANSYS V.11 (Ansys Inc., Canonsburg PA), el cual usa el método de volúmenes finitos, para la discretización espacial del dominio.

El método de integración temporal utilizado para las ecuaciones de cantidad de movimiento es el Second order backward Euler, y para el acoplamiento velocidad-presión se empleó el método SIMPLE, con un paso de tiempo de $0,01 \mathrm{~s}$, lo que permitió obtener resultados en intervalos de $1 / 100 \mathrm{~s}$. El tiempo total de simulación correspondiente a 6 veces al ciclo cardíaco. Los resultados utilizados se corresponden al último ciclo (sexto), donde se comprobó que éstos son independientes de las condiciones iniciales consideradas en las simulaciones $(U=V=W=0$ en $t=0$ s).

Las pruebas para la evaluación de la convergencia de las soluciones, fueron realizadas modificando básicamente los parámetros relacionados con el refinamiento de la malla en los lugares de interés para la dinámica del fluido (regiones de gran curvatura y efectos de pared), comprobándose que cuando la región de pared alcanza el valor de 1/3 del diámetro representativo de la arteria aorta, no se producen significativos cambios en los patrones de flujo. Se ha considerado paredes rígidas. Para mayor información, consultar (Vilalta et al., 2009).

\section{RESULTADOS Y DISCUSIÓN}

Es conocido que la dinámica del flujo sanguíneo en el interior del sistema circulatorio está gobernada por la interacción entre la pared arterial y el flujo sanguíneo, condicionando uno el comportamiento del otro.

Para el análisis del campo de flujo en el interior del saco aneurismático, se han seleccionado los modelos que representan los extremos de la asimetría, $\beta=0.2$ (mayor asimetría) y $\beta=0.8$ el cual es casi simétrico. Los tiempos estudiados se corresponden con los puntos de mayor interés dentro del ciclo cardíaco, $t=0.2 \mathrm{~s}, t=0.4 \mathrm{~s}, t=0.5 \mathrm{~s}$ y $t=0.7 \mathrm{~s}$. Estas regiones son las de inicio de la aceleración sístólica (1), la de máxima valor durante la sístole (2), un punto en la desaceleración sistólica (3) y un punto en la etapa diastólica (4). Se incluye también el análisis correspondiente a $\mathrm{t}=0 \mathrm{~s}$, por la importancia que tiene en la discusión de los resultados.

Los resultados se muestran en la Figura 3 , a través de la presentación de los vectores de velocidad resultante en el plano medio $y-z$, de la geometría.

Una característica importante de este análisis es que el campo de flujo está dominado por la presencia de vórtices en las regiones próximas de la pared del saco aneurismático. Del análisis integral del ciclo cardíaco (6 ciclos estudiados), se observa, al inicio de cada ciclo ( $\mathrm{t}=0 \mathrm{~s}$ ), la existencia de vórtices residuales dejados del ciclo anterior. A medida que disminuye la asimetría, se forma un chorro de flujo que atraviesa la región central del saco aneurismático, generando 2 núcleos de vórtices de menor tamaño que el existente cuando el aneurisma es más simétrico. Este comportamiento es típico hasta $\mathrm{t}=0.2 \mathrm{~s}$, aproximadamente. En este momento, ocurre un crecimiento de la región de recirculación, justo antes que se inicie la aceleración sistólica, y que llega a ocupar casi toda la región correspondiente al bulbo aneurismático, aumentando la intensidad, con el nivel de asimetría. De igual forma, en esta etapa existen vórtices de baja velocidad en el extremo distal del aneurisma, por la pared posterior. Esto resulta en vórtices que forman un lazo alrededor del núcleo de flujo. Los extremos proximal y distal están caracterizados por velocidades negativas en regiones cercanas a la pared, lo que se supone que es debido a los efectos convectivos que surgen como resultado del cambio de geometría. En la primera mitad de la sístole, el gradiente de presión eyecta vórtices aguas arriba, a medida que el flujo se va acelerando en el tiempo.

Las máximas velocidades y gradiente de velocidades son obtenidos en el pico máximo de velocidad, donde se forma un patrón casi simétrico en el saco aneurismático. La desaceleración temporal combinada con débiles efectos convectivos, son los factores dominantes en la segunda mitad de la sístole, donde chorros de alta velocidad, provocan significativas perturbaciones hemodinámicas, las que se inician en la etapa (3). 
A mitad del ciclo cardíaco, aproximadamente $t=0.6 \mathrm{~s}$, ocurre una reversión del flujo, causando una disminución de la intensidad de la recirculación del flujo y un movimiento del vórtice hacia el centro y aguas abajo del aneurisma. Esto resulta en una extensión de la estela del vórtice hacia la sección de entrada del AAA. Durante la diástole, el flujo recupera su dirección original con una ligera aceleración que provoca la traslación del vórtice principal hacia el extremo distal y la supresión de la estela. El final de esta fase, está caracterizada por un flujo casi constante y una intensificación de las perturbaciones debido al incremento de la velocidad local. Estos resultados son consistentes con otros de la literatura (Finol et al., 2003).
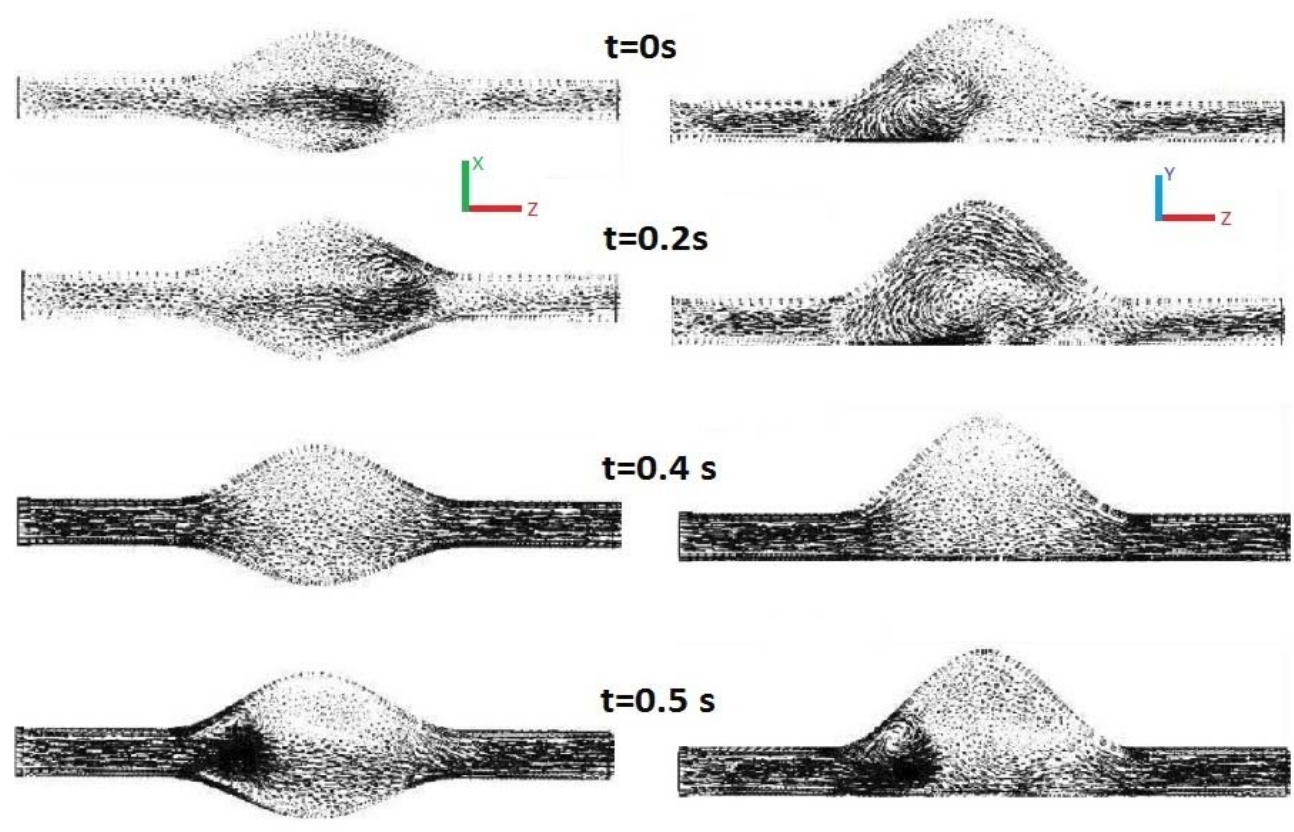

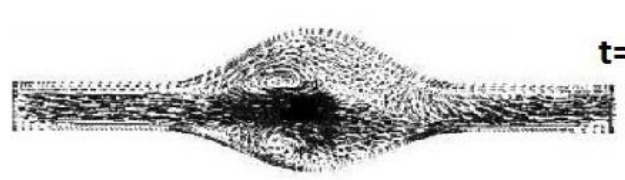

(a)

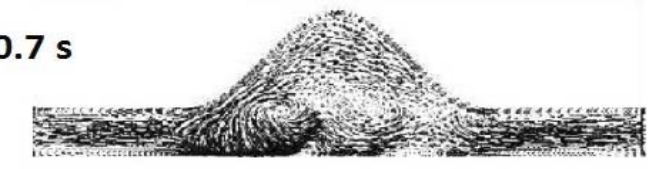

(b)

Fig. 3: Vectores velocidad que representan el campo de flujo en el interior de AAA, para valores de asimetría extremos: (a) $\beta=0.2$ y (b) $\beta=0.8$, en diferentes etapas del ciclo cardíaco.

\section{Tensiones tangenciales de pared provocadas por el campo de flujo}

La asimetría de los AAA, ejerce una gran influencia sobre la distribución de las tensiones tangenciales de pared. La Figura 4, muestra la distribución superficial de tensiones tangenciales para diferentes condiciones de asimetría $(\beta=0.8, \beta=0.6$ y $\beta=0.2$ y tiempos de ciclo cardíaco.

Para $t=0.2 \mathrm{~s}$, donde la curva de velocidad pulsátil está próxima a un punto de inflexión, las tensiones tangenciales presentan una distribución relativamente similar, siendo el rasgo más significativo la distorsión que provoca en este comportamiento los efectos causados por la imposición de un perfil de velocidades totalmente desarrollado como condición de entrada, lo cual ha sido reportado por otros autores. Esto se refleja con altos valores de tensión en el cuello de entrada al aneurisma. Justo después de la entrada al saco aneurismático, los patrones de velocidades provocan regiones de bajas tensiones, las que alcanzan sus valores menores a medida que se modifica la simetría del aneurisma. Aguas abajo de la sección de máximo diámetro, el extremo distal se caracteriza por patrones simétricos de flujo y regiones de recirculación las que inducen a gradientes de velocidad no uniformes y a un significativo incremento de las tensiones. Estas perturbaciones de flujo, diferencian el final de la diástole de un ciclo, de la sístole del siguiente, mientras que los vórtices remanentes de un ciclo permanecen en 
el inicio del siguiente. En las regiones de máximas tensiones tangenciales, éstas varían en hasta un $15 \%$ aproximadamente a medida que aumenta el grado de asimetría de los aneurismas.
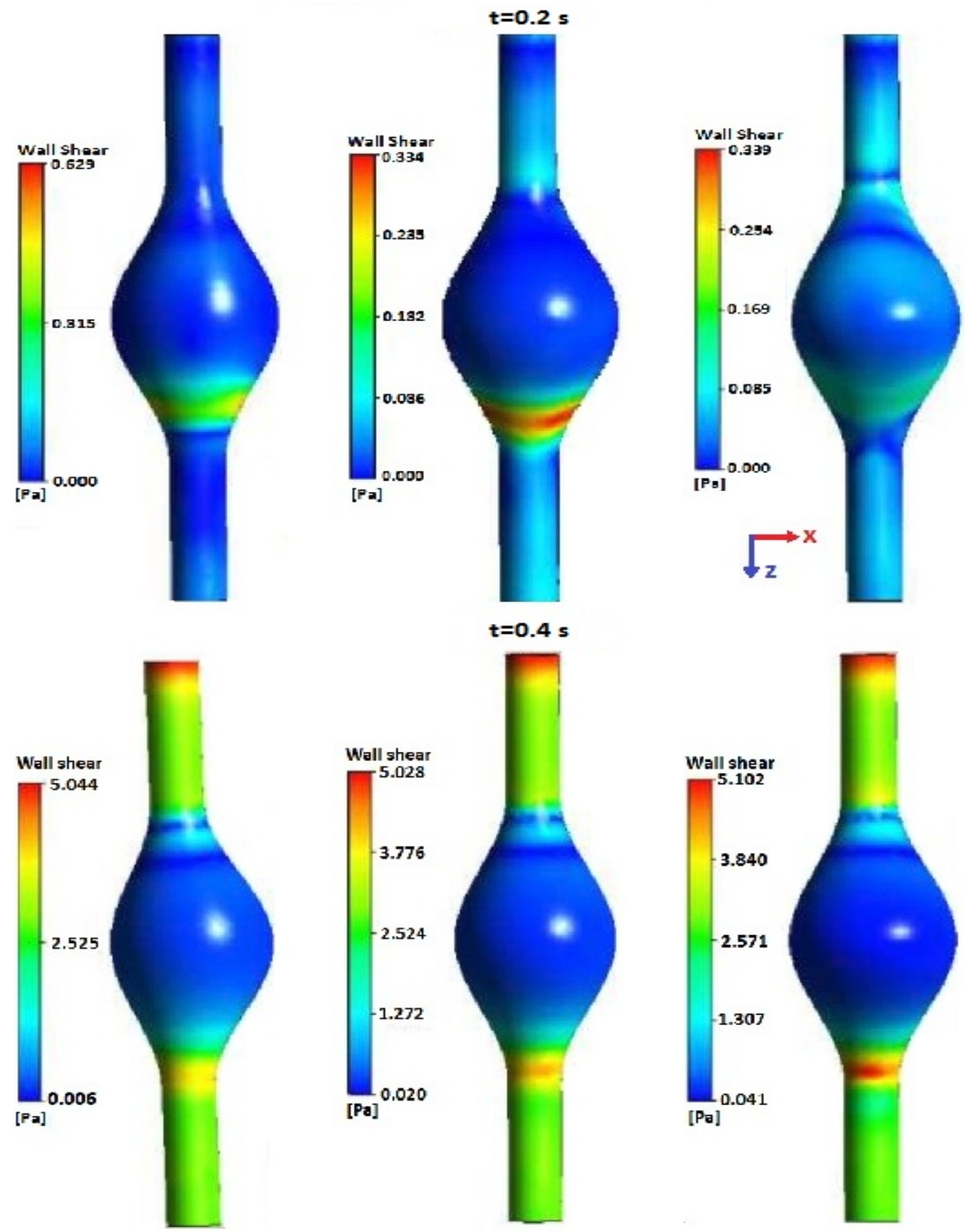

Fig. 4: Distribución de la tensión tangencial de pared para diferentes índices de asimetría y etapas del ciclo cardíaco. (a) $\beta=0.8$, (b) $\beta=0.6$ y (c) $\beta=0.2$.

Los máximos valores de tensiones tangenciales durante el ciclo cardíaco, se obtienen en la etapa correspondiente al máximo sistólico $(t=0.4 \mathrm{~s})$ donde, como ya fue analizado, las velocidades y los gradientes espaciales de ésta, son mayores. Los patrones de flujo completamente adheridos a la pared arterial, producen valores positivos de tensiones en todo el aneurisma cuyo patrón es aproximadamente uniforme hasta la sección media del aneurisma, independientemente del grado de asimetría. Esta uniformidad es obtenida tanto en la pared anterior como posterior. El análisis de la distribución de tensiones en el extremo distal muestra interesantes aspectos. Se debe tener en 
cuenta, como ya fue analizado, que esta región está caracterizada por patrones de flujo que se contraen a medida que la aceleración convectiva domina el flujo a fin de que pueda satisfacerse la conservación de la masa, lo cual provoca un incremento de las tensiones tangenciales y distribuciones asimétricas de ésta. Como resultado de esto, las regiones de ocurrencia de los máximos de tensiones tangenciales de pared, se obtienen, en el extremo distal de la pared anterior del aneurisma, como se indica en esta figura. Los incrementos de tensiones a medida que aumenta la asimetría de los aneurismas, están en el orden de $30 \%$. Las restantes etapas del ciclo cardíaco analizadas ( $\mathrm{t}=0.4 \mathrm{~s}$ y $\mathrm{t}=0.7 \mathrm{~s})$ muestran comportamientos similares cualitativamente, con incrementos de las tensiones a medida que aumenta la asimetría del orden de $17 \%$, en ambos casos.

\section{Factores biomecánicos asociados a la asimetría}

Como fue definido en la sección introductoria del presente trabajo, en la actualidad se presta gran atención a la definición de un nuevo enfoque para mejorar la predicción de la ruptura de AAA, el cual se basa en relaciones existentes entre factores de diferentes naturalezas y escalas: factores o determinantes biomecánicos.

A continuación se presentan los resultados obtenidos de la aplicación del método propuesto (Vilalta et al, 2011b) para evaluar el riesgo de ruptura de los AAA, utilizando apenas los biodeterminantes geométricos, o sea, aquellos resultantes de la combinación de los parámetros geométricos que caracterizan el aneurisma. Dos cuestiones son definidas a fin de justificar la aplicación del método y la validez de los resultados a partir de geometrías idealizadas. La primera es que el valor de los parámetros geométricos utilizados para generar el modelo, está en los rangos fisiológicos de esta patología. Por lo tanto, es perfectamente posible encontrar un aneurisma real con dimensiones semejantes a las utilizadas aquí. La segunda, es que, como la investigación desarrollada debe aportar información para mejorar la predicción del riesgo de ruptura, la precisión de los resultados es coherente con las escalas de tiempo típicas de esta patología.

La hipótesis desarrollada por los autores, es que como el AAA se define conceptualmente como una deformación localizada y progresiva de la aorta, sus parámetros geométricos pueden caracterizar el mismo desde el punto de vista geométrico y está bien documentado (Bernad et al., 2009) que la geometría del AAA tiene una fuerte influencia en su potencial de ruptura. Los principales parámetros geométricos (definidos en la Fig (1)), fueron adecuadamente relacionados para definir los biodeterminantes geométricos (BDGs): Tasa de deformación $\chi$, Índice de asimetría $\beta$, Índice de dilatación $\gamma$, Razón superficial AAA/TIL (tombo intraluminal) $\lambda$, Tasa de crecimiento $\varepsilon \mathrm{y}$ Espesor relativo $l$.

El método se resume a continuación. Una vez definidos los biodeterminantes, éstos fueron clasificados en intervalos los cuales están relacionados con el nivel de riesgo ponderado, NRPi, que evalúa el peso de cada intervalo en las posibilidades de ruptura. Igualmente, los DBs tienen asociado un coeficiente de ponderación que considera el peso de cada determinante en el riego de ruptura, $\omega i$. Los valores de NRP para cada DB (i), fue obtenido de información disponible en la literatura abierta y consultada con especialista. Los valores relativos a $\omega i$, fueron definidos teniendo en cuenta los resultados presentados en Vaquero, 2009.

Conjugando los DBs, el NRPi y el $\omega i$, se ha definido el Índice de Ruptura $(I R(t))$, de acuerdo con la Ecuación (7) y cuyo valor está entre 0 y 1.

$$
I R(t)=\sum_{i=1}^{6} \omega_{i} B F_{i}
$$

Para conocer el grado de desarrollo del aneurisma y las posibilidades de ruptura, se han establecidos 4 rangos. Si $I R(t)<0.2$, el riesgo que presenta el aneurisma es muy bajo, no sugiriéndose ninguna acción. Cuando está en el rango entre 0.2 a 0.45 , el riesgo de ruptura es bajo, por lo que el paciente debe ser mantenido bajo observación. Cuando el valor es mayor de 0.45, el facultativo médico debe considerar someter al paciente a una reparación electiva. Si se 
alcanza el valor de 0.7 o mayor, se considera que el aneurisma está en zona de peligro, la ruptura puede ocurrir en cualquier momento y debe procederse a la intervención quirúrgica de inmediato. También se recomienda que, cuando el valor de $I R(t)$ sea mayor que 0.45 , se consideren otros síntomas que pueda presentar el paciente como dolores abdominales y de espalda, vómitos etc los que pueden ser indicativos de ruptura.

Una aspecto importante del método es que, en el caso de que no se disponga de algún dato relativo a los parámetros geométricos, se produce un ajuste automático del algoritmo, para emplear en el cálculo del $I R(t)$ sólo los BDGs asociados a los parámetros disponibles, ponderando el resultado final de acuerdo a los parámetros geométricos que emplea.

Teniendo en cuenta la metodología propuesta en ese trabajo, se realizará a continuación la caracterización geométrica del aneurisma, calculándose los DBGs asociados y el estado general de riesgo de ruptura. Debe considerarse que, al ser la asimetría el único biodeterminante que cambia, existirán los 4 modelos a evaluar.

Como los parámetros geométricos definidos son: diámetro máximo $D$, longitud $L$, asimetría $\beta$ y el diámetro de la artería infrarenal $d$, los biodeterminantes calculados en este caso son: Tasa de deformación $\chi$, Índice de asimetría $\beta$ e Índice de dilatación $\gamma$. Otro biodeterminante que es incluido en el estudio, fue la tasa de crecimiento, $\varepsilon$. En la práctica clínica, este factor se define como el crecimiento del diámetro máximo entre dos revisiones sucesivas. Cuando no existe dato previo, Bernstein et al., 1984, basado en datos clínicos, sugieren una correlación para la tasa de expansión del diámetro, válida para $D>45 \mathrm{~mm}$, de la forma

$$
\dot{d}_{A A A}=0.0037 D_{A}^{3}-0.04 D_{A}^{2}-0.0317 D_{A}+0.705 \text { (cm/año) }
$$

donde $D_{A}$ es el diámetro máximo del aneurisma en el estado actual.

Así, el diámetro máximo previo es:

$$
D_{P}=D_{A}-\dot{d}_{A A A} \Delta t
$$

siendo $\Delta t$, el tiempo entre dos revisiones consecutivas. Este valor, de acuerdo con la práctica médica actual, es típicamente 1 año. De esta forma, la tasa de crecimiento se determina por

$$
\varepsilon=\frac{D_{A}-D_{P}}{\Delta T}(\mathrm{~cm} / \mathrm{año})
$$

La Tabla 1 muestra un resumen de los resultados obtenidos que permite, en primer término, hacer una valoración integral del estado de desarrollo de este aneurisma.

\begin{tabular}{|c|c|c|c|c|c|c|c|c|c|c|c|c|c|}
\hline \multirow{3}{*}{ Modelo } & \multicolumn{12}{|c|}{ Biodeterminantes } & \multirow{3}{*}{$\mathrm{RI}(\mathrm{t})$} \\
\hline & \multicolumn{3}{|c|}{ Tasa de deformación } & \multicolumn{3}{|c|}{ Asimetría } & \multicolumn{3}{|c|}{ Índice de dilatación } & \multicolumn{3}{|c|}{ Tasa de crecimiento } & \\
\hline & Valor & NRP & $\omega \mathrm{i}$ & Valor & NRP & $\omega \mathrm{i}$ & Valor & NRP & $\omega \mathrm{i}$ & Valor & NRP & $\omega \mathrm{i}$ & \\
\hline 1 & 3 & 0.7 & 0.35 & 0.2 & 1 & 0.07 & 0.5 & 1 & 0.1 & 0.52 & 1 & 0.34 & 0.81 \\
\hline 2 & 3 & 0.7 & 0.35 & 0.4 & 1 & 0.07 & 0.5 & 1 & 0.1 & 0.52 & 1 & 0.34 & 0.81 \\
\hline 3 & 3 & 0.7 & 0.35 & 0.6 & 0.7 & 0.07 & 0.5 & 1 & 0.1 & 0.52 & 1 & 0.34 & 0.78 \\
\hline 4 & 3 & 0.7 & 0.35 & 0.8 & 0.1 & 0.07 & 0.5 & 1 & 0.1 & 0.52 & 1 & 0.34 & 0.74 \\
\hline
\end{tabular}

Tabla 1: Comportamiento individual de los DBGs relacionados con la geometría para cada uno de los cuatro modelos estudiados.

En general, se observa que los cuatro modelos de aneurismas presentan $I R(t)$ en el rango característico de la zona peligrosa $(>0.7)$, La tendencia es que a medida que disminuye el valor 
del coeficiente de asimetría (el aneurisma es más asimétrico), aumenta el peligro de ruptura. Este resultado está teóricamente fundamentado y ha sido reportado en estudios previos (Biasetti et al. 2010, Li y Kleinstreuer, 2007).

Del análisis individual de los biodeterminantes geométricos, se puede observar que la tasa de deformación presenta un valor catalogado como de alto riesgo, dado fundamentalmente por el valor del diámetro aórtico infrarenal $d$, el cual es un valor ligeramente inferior al valor medio típico de la población que está entre 1.9-2.6 cm (Myers et al. 2001). Referente a $D$, es un valor de riesgo dentro la práctica clínica, aunque con frecuencia la literatura y los registros clínicos, hacen referencia a valores mayores, sin que ocurra la ruptura.

De acuerdo con la definición del Índice de dilatación, es de esperar que para las geometrías analizadas, este factor no sea muy influyente pues la longitud es relativamente grande. Por lo tanto, el efecto que produce la deformación arterial es menor, al estar distribuida en una porción mayor de arteria.

El valor obtenido para la tasa de crecimiento está justificada por la reconocida dependencia entre la tendencia del crecimiento de los aneurismas con el diámetro. Con el aumento del diámetro ocurre un cambio en la tendencia de crecimiento pasando de crecer de forma linear a exponencial (Dryjski et al, 1994). Por esto es de esperarse que aneurismas con diámetro máximo del orden de $60 \mathrm{~mm}$, tengan una alta tasa de crecimiento.

La asimetría, como factor biomecánico, es un valor impuesto aunque con diferentes grados de riesgo. Cuando se considera dentro del conjunto de DBGs, se observa que de valores de más riesgo al de menor riesgo de asimetría, el valor del $I R(t)$ sólo disminuye un $7 \%$, manteniéndose siempre dentro de la categoría de aneurismas en peligro de ruptura. Este resultado confirma que la asimetría es de los DBGs con menor influencia en el riesgo de ruptura de aneurismas de aorta abdominal, lo cual es coincidente con el enfoque propuesto por Kleinstreuer y Li, 2006.

\section{CONCLUSIONES}

La dinámica de las estructuras de vórtices, están bien caracterizadas en las etapas del ciclo cardíaco y modelos geométricos asimétricos estudiados. Esta puede ser resumida, a partir de considerar la existencia de vórtices residuales del ciclo anterior, en el actual ciclo. Al inicio de la aceleración sistólica, ocurre un movimiento de los vórtices (residuales) hacia la región de la pared anterior del aneurisma, acompañados estos con zonas de recirculación dominantes en el saco aneurismático. Con el aumento de la velocidad del flujo sanguíneo, etapa 2, los vórtices forman un lazo alrededor del núcleo del chorro que atraviesa el aneurisma lo que provoca la eyección de los vórtices aguas arriba. En etapas siguientes, estas estructuras se desplazan hacia el centro y hacia la región del extremo distal del aneurisma, ejerciendo una importante influencia sobre la pared anterior del extremo distal. Este comportamiento se ve igualmente reflejado en la distribución de tensiones tangenciales, las cuales muestran sus mayores valores en esta región, los que se incrementan con el aumento del grado de asimetría.

Los aneurismas de aorta abdominal son, en esencia, asimétricos debido a las restricciones a su expansión que imponen la columna vertebral y otros órganos. No obstante a eso, este trabajo ha confirmado que entre los determinantes geométricos, la asimétria no es de los factores que más influyen en su potencial de ruptura.

\section{AGRADECIMIENTOS}

Los autores de este trabajo agradecen al Ministerio de Ciencia e Innovación de España y a la Junta de Castilla y León, el apoyo financiero para la realización del presente trabajo. También agradecen la inestimable colaboración del Dr. Carlos Vaquero Puerta, del Hospital Clínico Universitario de Valladolid, por la revisión del trabajo y sus apreciaciones sobre este tema.

\section{REFERENCIAS}

Bernard, S.I., y otros 4 autores, Effects of asymmetry in patient-specific wall shear stress analyses of abdominal aortic aneurysm. Journal of Chinese Clinical Medicine. 41(8), 421-429 (2009). 
Bernstein, E., Chan, E., Abdominal aortic aneurysm in high-risk patients. Outcome of selective management based on size and expansion rate. Annals of Surgery. 200, 255-263 (1984).

Biasetti, J., y otros 4 autores, Hemodynamics of normal aorta compared to fusiform and saccular abdominal aortic aneurysm with emphasis on a potential thrombus formation mechanism. Annals of Biomedical Engineering, 38(2), 380-390 (2010).

Di Martino, E.S., y otros 6 autores, Fluid-structure interaction within realistic three-dimensional models of the aneurysmatic aorta as a guidance to assess the risk of rupture of the aneurysm, Medical Eng \& Physics: 23, 647-655 (2001).

Dryjski, M., y otros 7 autores, The small abdominal aortic aneurysm: the eternal dilemma. J. Cardiovascular Surgery, 35 95-100 (1994).

Ene, F., y otros 6 autores, In vitro evaluation of the effects of intraluminal thrombus on abdominal aortic aneurysm wall dynamic. Medical Engineering \& Physics, 33, 957-976 (2011).

Fillinger, M.F., Marra, S.P., Raghavan, M.L., Kennedy, F.E., Prediction of rupture in abdominal aortic aneurysm during observation: Wall stress versus diameter, J. Vasc Surg: 37, 724-32 (2003).

Finol, E.A., Amon, C.H., Flow-induce wall shear stress in abdominal aortic aneurysms: Part II pulsatile flow hemodynamics. Computer Methods in Biomechanics and Biomechanical Engineering, 5:329-328, (2002).

Finol, E.A., Keyhani, K., Amon, CH. The effect of asymmetry in abdominal aortic aneurysm under physiologically realistic pulsatile flow conditions, Journal of Biomechanical Engineering: 125(2), 207-212 (2003).

Gasser, T.C., y otros 4 autores, Biomechanical rupture risk assessment of abdominal aortic aneurysms: Model Complexity versus predictability of finite element simulations. Eur J Vasc Endovasc Surg, 40, 176-185 (2010).

Georgakaratos, E., y otros 6 autores, The role of geometric parameters in the prediction of abdominal aortic aneurysm wall stress. Eur. J. Vasc Endovasc Surg, $3942-48$ (2010).

Giannoglou, G., y otros 7 autores, Predicting the risk of rupture of abdominal aortic aneurysms by utilizing various geometrical parameters: revisiting the diameter criterion. Angiology 57(4) 487-494. (2006).

Kleinstreuer, C., Li, Z., Analysis and computer program for rupture-risk prediction of abdominal aortic aneurysms. BioMedical Engineering OnLine, 5:19, (2006).

Li, Z., Kleinstreuer, C., A comparision between different asymmetric abdominal aortic aneurysm morphologies employing computational fluid-structure interaction analysis. Eur. J. Mech B/Fluids 26, 615-631 (2007).

Maier, A., y otros 5 autores. A comparison of diameter, wall stress, and rupture potential index for abdominal aortic aneurysm rupture risk prediction. Annals of Biomedical Engineering, 38(10), 3124-3134, (2010).

McGloughlin, T.M., Doyle, B., New approaches to abdominal aortic aneurysm rupture risk assessment: engineering insights with clinical gain. Arteriosclerosis thrombosis and vascular biology, 30(9), 1687-1694, (2010).

Mills, C., y otros 5 autores, Pressure-flow relationships and vascular impedance in man. Cardiovascular Res. 4, 405-417 (1970).

Milnor, W., Hemodynamics. 2nd Edition. Williams and Wilkins, Baltimore. MD, p. 34-35, (1989). 
Moore, J.E., Ku. D.N., Pulsatile velocity measurements in a model of the human abdominal aorta under resting conditions. ASME Journal of Biomechanical Engineering, 116:337-346, (1994).

Myers, K., Devine, T., Barras, C., Self G. Endoluminal versus open repair for abdominal aortic aneurysm. (2001). Disponible en internet: http://www.fac.org.ar/scvc/llave/interven/myers/myersi.html.

Papaharilaou, Y., Ekaterinaris, J., Manousaki, E., Katsamouris, AN. A decoupled fluid structure approach for estimating wall stress in abdominal aortic aneurysm, Journal of Biomechanics: 40, 464-75 (2007).

Vaquero, C., Factores geométricos y su incidencia en Aneurismas de Aorta Abdominal. Estudio preliminar". Reporte interno 33/09 del Hospital Clínico de Valladolid. (2009).

Vilalta, G., Nieto, F., Rodríguez, M., Lipsa, L.M., O’Connor, J., Dounié, O., Influencia de la geometría de aneurismas de aorta abdominal en la dinámica del flujo sanguíneo y en su riesgo de ruptura. Ingeniería Mecánica 12(2) 25-36 (2009).

Vilalta, G., Nieto, F., Vaquero, C., Vilalta, J.A. Quantitative Indicator of Abdominal Aortic Aneurysm Rupture Risk Based on its Geometric Parameters, World Academy of Science, Engineering and Technology: 70, 181-185, (2010).

Vilalta, G., Nieto, F., Vaquero, C., Vilalta, J.A., Pérez, M.A., Patient-specific Clinical Assessment of Abdominal Aortic Aneurysm Rupture Risk Based on its Geometric Parameters, In Proceedings of the Eighth International Conference on Biomedical Engineering, BioMed 2011, Innsbruck-Austria, Feb. 16-18 (2011a).

Vilalta, G., Nieto, F., Vaquero, C., Vilalta, J.A., Lipsa, L.M., Gutierrez, V., A Method for Patientspecific Assessment of Abdominal Aortic Aneurysm Rupture Risk Based on Geometric Biomechanics Factors. Clinical Validation Test. En Proceedings of the V Latinamerican Congress on Biomedical Engineering, CLAIB 2011, La Habana-Cuba, May. 18-20 (2011b). 